\title{
Computation of eigenvectors corresponding to multiple eigenvalues
}

\begin{abstract}
A. L. Andrew and G.C. Elton
This paper examines a class of common numerical methods for computing eigenvectors of a compact linear operator. A necessary and sufficient condition is established for every element of an arbitrary given eigenspace to be the limit of a sequence of the approximate eigenvectors obtained by any given method in this class.
\end{abstract}

This paper studies convergence of approximate operator methods [1] (which include all projection methods as special cases) for numerical computation of the eigenvectors of a compact linear operator in Hilbert space. For the sake of generality and simplicity, results are proved in an abstract setting. Application of results of this type to specific numerical methods and to differential equations is described in [1].

In the case of simple nonzero eigenvalues, approximate operator methods are known to be convergent for both eigenvalues and eigenvectors. In the case of a (nonzero) multiple eigenvalue it is known that, although the sequence $\left\{\psi_{n}\right\}$ of approximate eigenvectors is not always convergent, the component of $\psi_{n}$ outside the corresponding eigenspace converges to zero [1]. In the case of projection methods, Vaĭnikko [3] has proved a quantitative version of this result. However Pol'ski ' [2] has shown, by means of an example, that a compact linear operator may have an eigenvector which is not a limit point of any sequence of approximate eigenvectors obtained by a projection method.

This paper establishes a necessary and sufficient condition for every 
element of a given eigenspace of a compact linear operator to be the limit of a sequence of the approximate eigenvectors obtained by an approximate operator method. Note that, by definition of $U_{n}$ below, $\operatorname{dim} U_{n}$, the dimension of $U_{n}$, satisfies $1 \leq \operatorname{dim} U_{n} \leq m$ for all $n$. Hence Theorem 1 of [1] is the special case $m=1$ of the theorem proved below.

THEOREM. Let $T_{n}(n=1,2, \ldots)$ and $T$ be compact linear operators, mapping a (real or complex) Hilbert space $H$ into itself. Let $E$ be the set of all unit vectors in $H$. Let $\mu$ be a nonzero eigenvalue of $T$ and let the corresponding eigenspace $V$ have dimension $m$. For each positive integer $n$, let $\mu_{n}$ be an eigenvalue of $T_{n}$ with corresponding eigenspace $V_{n}$. Let

$$
\left\|T_{n}-T\right\| \rightarrow 0 \text { and }\left|\mu_{n}-\mu\right| \rightarrow 0 \text { as } n \rightarrow \infty \text {. }
$$

For all $n$ for which $\operatorname{dim} V_{n}>m$ let $U_{n}$ be an arbitrary given m-dimensional subspace of $V_{n}$. For all other $n$ let $U_{n}=v_{n}$. Then the following conditions are equivalent:

(a) for all but finitely mony $n, \operatorname{dim} V_{n} \geq m$;

(b) for all $\psi$ in $V \cap E$, there is a sequence $\left\{\psi_{n}\right\}$ with $\psi_{n}$ in $U_{n} \cap E$ and $\psi_{n} \rightarrow \psi$ as $n \rightarrow \infty$;

(c) for all $\psi$ in $V \cap E$, there is a sequence $\left\{\psi_{n}\right\}$ with $\psi_{n}$ in $V_{n} \cap E$ and $\psi_{n} \rightarrow \psi$ as $n \rightarrow \infty$.

Proof. $(a) \Rightarrow(b)$. Suppose the contrary. Then there must be an infinite strictly increasing sequence $S$ of positive integers such that

$$
(\exists a>0)(\forall n \in S)\left(\forall \psi_{n} \in U_{n} \cap E\right)\left\|\psi_{n}-\psi\right\|>d \text {. }
$$

By (a), $S$ contains an infinite subsequence $S_{0}$ such that for each $n$ in $S_{0}, U_{n}$ contains an m-tuple $\left\{\psi_{n I}, \ldots, \psi_{n m}\right\}$ of orthonormal vectors. Since $T$ is compact, the image of any sequence of vectors from $E$ must contain a convergent subsequence. Since $m$ is finite (as $T$ is compact), a simple inductive argument on the components of the above m-tuples yields 
an infinite subsequence $S_{1}$ of $S_{0}$ such that

$$
\mu^{-1} T \psi_{n i} \rightarrow \psi_{0 i}, \quad i=1, \ldots, m
$$

when $n$ is restricted to $S_{1}$ (which is independent of $i$ ). Restricting $n$ to $S_{1}$ gives

$$
|\mu|\left\|\psi_{0 i}-\psi_{n i}\right\| \leq\left\|\mu \psi_{0 i}-T \psi_{n i}\right\|+\left\|T-T_{n} i\right\| \psi_{n i}\left\|+\left|\mu_{n}-\mu\right|\right\| \psi_{n i} \| \rightarrow 0 \text { as } n \rightarrow \infty
$$

by (1) and (3). Hence

$$
\psi_{n i} \rightarrow \psi_{0 i} \quad\left(n \in S_{1}\right) \quad i=1, \ldots, m \text {. }
$$

Since $T$ is continuous, and, for each $n,\left\{\psi_{n 1}, \ldots, \psi_{n m}\right\}$ is an orthonormal set, (3) and (4) show that $\left\{\psi_{01}, \ldots, \psi_{0 m}\right\}$ forms an orthonormal basis for $V$. Hence there exist constants $c_{1}, \ldots, c_{m}$ such that $\psi=\sum_{i=1}^{m} c_{i} \psi_{0 i}$. Define $\psi_{n}=\sum_{i=1}^{m} c_{i} \psi_{n i}$ for $n \in S_{1}$. Clearly $\psi_{n} \in U_{n} \cap E$ and from (4)

$$
(\forall a>0)\left(\exists n \in S_{1}\right)\left(\bar{\exists} \psi_{n} \in U_{n} \cap E\right)\left\|\psi_{n}-\psi\right\|>d .
$$

This contradicts (2), as $S_{1} \subseteq S$. The result follows,

$$
\begin{aligned}
& \text { (b) } \Rightarrow \text { (c). Trivial, as } U_{n} \subseteq V_{n} \text {. } \\
& \text { (c) } \Rightarrow \text { (a). Let } \psi_{01}, \ldots, \psi_{O m} \text { be } m \text { orthonormal vectors in } V \text {. }
\end{aligned}
$$

Then, by $(c)$, there are $m$ sequences $\left\{\psi_{n i}\right\}, i=1, \ldots, m$, each with its $n$-th element in $V_{n} \cap E$, and with $\psi_{n i} \rightarrow \psi_{0 i}$ as $n \rightarrow \infty$. It is. sufficient to prove that, for sufficiently large $n$, none of the $m$ vectors $\psi_{n i}(i=1, \ldots, m)$ is a linear combination of the others. Suppose the contrary. Then without loss of generality we may suppose $\psi_{n 1}=\sum_{i=2}^{m} c_{i} \psi_{n i}$ where $\left|c_{i}\right| \leq 1, i=2, \ldots, m \cdot$ Now

$$
\begin{aligned}
\left|\left(\psi_{n i}, \psi_{n j}\right)\right| \leq\left|\left(\psi_{0 i}, \psi_{0 j}\right)\right|+\left|\left(\psi_{n i}-\psi_{0 i}, \psi_{0 j}\right)\right| & +\left|\left(\psi_{0 i}, \psi_{n j}-\psi_{0 j}\right)\right| \\
& +\left|\left(\psi_{n i}-\psi_{0 i}, \psi_{n j}-\psi_{0 j}\right)\right|<1 / m
\end{aligned}
$$


for $i \neq j$ and $n$ sufficiently large (since $\left(\psi_{0 i}, \psi_{0 j}\right)=0$ and $\left.\psi_{n i} \rightarrow \psi_{0 i}\right\}$.

Hence, for $n$ sufficiently large,

$I=\left(\psi_{n \perp}, \psi_{n \perp}\right)=\sum_{i=2}^{m} c_{i}\left(\psi_{n i}, \psi_{n \perp}\right) \leq \sum_{i=2}^{m}\left|c_{i}\right|\left|\left(\psi_{n i}, \psi_{n \perp}\right)\right|<(m-1) / m<1$.

The result follows.

COROLLARY. The theorem remains true when $(a),(b)$ and $(c)$ are replaced by $\left(a^{\prime}\right),\left(b^{\prime}\right)$ and $\left(c^{\prime}\right)$ below.

(a'). For infinitely many $n, \operatorname{dim} V_{n} \geq m$.

(b'). [respectively (c')]. For alz $\psi$ in $V \cap E$ there is a sequence $\left\{\psi_{n}\right\}$ with $\psi_{n}$ in $U_{n} \cap E$ [respectively $V_{n} \cap \bar{E}$ ] such that $\psi$ is a limit point of $\left\{\psi_{n}\right\}$.

Proof. $\left(a^{\prime}\right) \Rightarrow\left(b^{\prime}\right)$. From the sequence $\left\{V_{n}\right\}$ delete those terms with $\operatorname{dim} V_{n}<m$ and apply the theorem.

$\left(b^{\prime}\right)=\left(c^{\prime}\right)$. Trivial, as before.

$\left(c^{\prime}\right)=\left(a^{\prime}\right)$. The sequence $\left\{\psi_{n}\right\}$ contains a subsequence converging to $\psi$. Apply the theorem to this subsequence.

\section{References}

[1] A.L. Andrew, "Convergence of approximate operator methods for eigenvectors", Bull. Austral. Math. Soc. 3 (1970), 199-205.

[2] N.1. Pol'skiY, "On the convergence of certain approximate methods of analysis" (Russian), Ukrain. Math. $\bar{z} .7$ (1955), 56-70.

[3] G.M. Vainnikko, "Asymptotic evaluations of the error of projection methods for the eigenvalue problem" (Russian), $\check{z}$. Vycisl. Mat. $i$ Mat. Fiz. 4 (1964), 405-425. English trans1. USSR Comput. Math. and Math. Phys. 4 (1964), no. 3, (1966), 9-36.

La Trobe University,

Bundoora, Victoria. 